CASE REPORT

\title{
A novel presentation of juvenile idiopathic arthropathy
}

\author{
L Dyet, C Pilkington, A Raffles
}

Arch Dis Child 2003;88:1015-1016

$\mathrm{T}$ oe-walking is frequently observed in general paediatrics. Causes include habit, congenital Achilles tendon shortening, cerebral palsy, and rarer aetiologies. ${ }^{1}$ Correct diagnosis of the underlying aetiology has implications for management. A benign aetiology can often be excluded if toe-walking is acquired, ${ }^{2}$ or affects balance or gait pattern. ${ }^{3}$ We report a case of juvenile idiopathic arthropathy presenting as toe-walking. This reminds clinicians to consider arthropathies in the differential diagnosis of toe-walking.

\section{CASE REPORT}

A $3 \frac{1}{2}$ year old white girl was referred to a general paediatric clinic with a six month history of an abnormal gait. The child had been born at full term with no complications and developmental progress was normal to date. She had walked independently at 11 months and had been able to run normally from the age of 18 months. A gait abnormality was first noted at 3 years of age when there were intermittent episodes of toe-walking. This had subsequently become permanent. Her parents had encouraged her to walk normally, but despite their efforts the problem had worsened to the point that she was unable to run or climb stairs.

At first presentation it was established that the child was now having difficulty in balancing, and appeared to grip the carpet with her toes when walking (see fig 1). She complained of persistent leg pains that improved with simple analgesia, but denied aching or painful joints. Her parents described her as being increasingly lethargic, but there was initially no obvious cause for this.

Clinical examination revealed the patient to be on the 91st centile for height and weight, although accurate measurement of height was difficult as she had fixed plantar flexion deformities and limited dorsiflexion of both ankles. There was no pain or swelling in her ankles at this time. Examining her gait she walked on her toes with her knees flexed, and was unable to place her feet flat on the ground without loss of balance. Examination of other joints revealed no deformity, swelling, pain, or erythema at any other sites. Her development was normal apart from her gait abnormality.

The patient was referred for physiotherapy assessment, and investigations were requested. These included a plain spinal $x$ ray examination and a magnetic resonance scan of her brain and spine, both of which were normal.

A month after her original presentation the child's parents reported a gradual onset of swelling of the child's wrists, knees, and ankles. Clinical assessment revealed that she had developed fixed flexion deformities of both knees with effusions at the knees, wrists, and ankles. Movements at the ankle remained limited. There was no evidence of iritis and she remained systemically well.

Erythrocyte sedimentation rate at this time was measured at $67 \mathrm{~mm} / \mathrm{h}$ and serum $\mathrm{C}$ reactive protein level was raised at $11 \mathrm{mg} / \mathrm{l}$. An autoantibody screen was negative. A diagnosis of polyarticular juvenile idiopathic arthritis was made and she was referred to a tertiary rheumatology centre for further assessment.

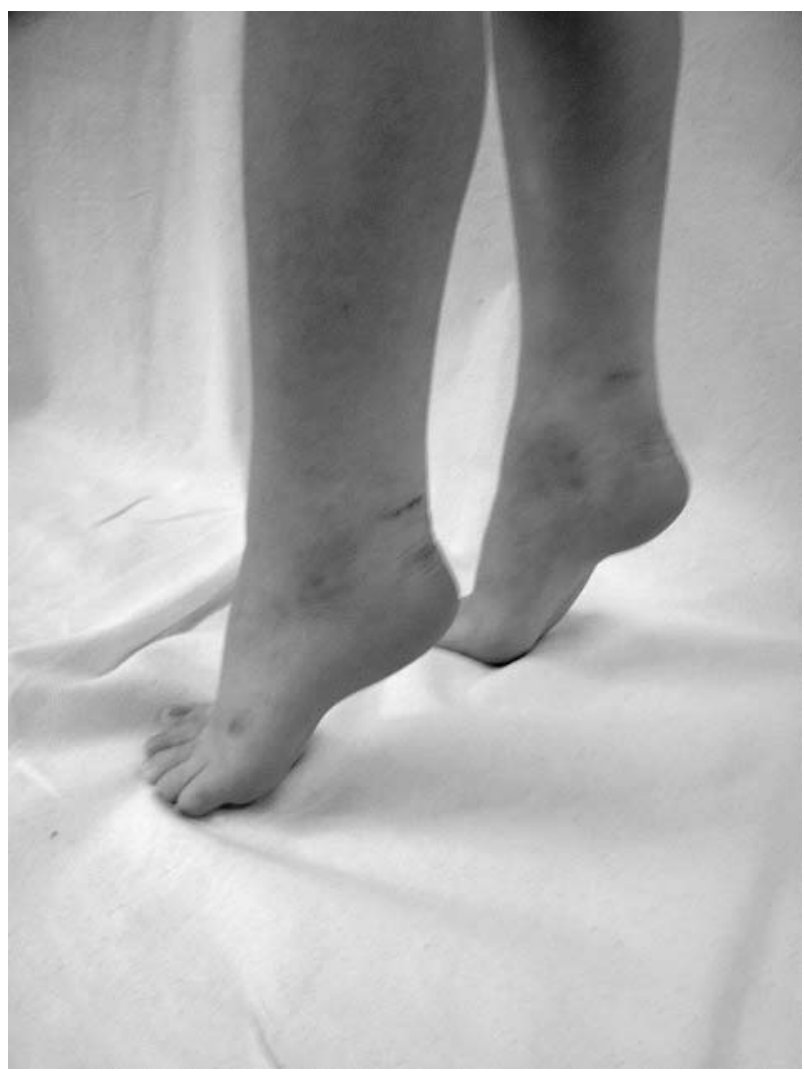

Figure 1 Toe-walking.

She was treated with high dose pulsed intravenous methylprednisolone because she had more than four joints involved in her disease. Following this treatment her range of movement in all affected joints improved significantly; in particular her ankles became freely mobile. She regained independent mobility; however, she still tended to toe-walk but generally her gait had improved. Following the pulsed steroids, weekly oral methotrexate was commenced.

\section{DISCUSSION}

Toe-walking is a common problem that presents to general paediatric outpatient clinics. The majority of cases are due to "habitual toe-walking", this usually being a diagnosis of exclusion, or alternatively due to congenital shortening of the Achilles tendon. A review of the literature reveals a smaller proportion of cases to be related to spastic or diplegic cerebral palsy, neuromuscular disease, spinal dysraphism, or pervasive developmental disorders. ${ }^{4}$ Accurate diagnosis has important implications for long term management and prognosis. Treatment can involve physiotherapy, occupational therapy, and where appropriate surgery to lengthen a shortened Achilles tendon. 


\section{Learning points}

- In acquired toe-walking, look for a diagnosis

- Remember to fully examine hips and knees for evidence of arthropathy

- Check inflammatory markers prior to arranging investigations such as MRI or $x$ ray examination to look for structural disorders

- Young children with juvenile idiopathic arthritis may not have joint pain or swelling at presentation

For this particular child a presumptive diagnosis of habitual toe-walking was made while awaiting the results of further investigations. In the interim progressive disease made the diagnosis more apparent. In retrospect there were some features not typical of habitual toe-walking at presentation. In particular the observation that she did not start walking abnormally until 3 years of age. Habitual toewalking usually occurs from the initiation of independent walking. ${ }^{2}$ Affected individuals usually walk with a normal well coordinated gait pattern without loss of balance. ${ }^{3}$ This patient was having problems with balance and was having difficulty running and walking up stairs. However, on initial presentation she had no joint swelling or pain, although a mild degree of fixed flexion deformity at her knees may have been missed.

Juvenile idiopathic arthropathy can present in a manner that may not initially suggest the diagnosis of a chronic arthritis. It is a recognised cause of failure of motor development, ${ }^{6}$ but the paediatric and rheumatology literature do not describe presentations with toe-walking. It is, however, recognised that toe-walking is more often seen in juvenile idiopathic arthritis in those with inflammation of the Achilles tendon insertion or with heel fasciitis. As toewalking is a relatively common presenting problem to primary and specialist paediatric care, it is important to consider an arthropathy in the differential diagnosis, especially if there are atypical features.

\section{Authors' affiliations}

L Dyet, A Raffles, Child Health Department, Queen Elizabeth II Hospital, Welwyn Garden City, Herts AL7 4HQ, UK

C Pilkington, Hospital for Sick Children, Great Ormond Street, London $\mathrm{WCl}$, UK

Correspondence to: $\operatorname{Dr}$ A Raffles, Consultant Paediatrician, Child Health Department, Queen Elizabeth II Hospital, Welwyn Garden City, Herts AL7 $4 \mathrm{HQ}$, UK; rafflesdr@btinternet.com

Accepted 9 March 2003

\section{REFERENCES}

1 Furrer F, Deonna T. Persistent toe-walking in children. A comprehensive clinical study of 28 cases. Helv Paediatr Acta 1982;37:301-16.

2 Shulman LH, Sala DA, Chu MLY, et al. Developmental implications of idiopathic toe walking. J Pediatr 1997; 130:541-6.

3 Caselli MA, Rzonca EC, Lue BY. Habitual toe-walking: evaluation and approach to treatment. Clin Podiatr Med Surg 1988;5:547-59.

4 Sala DA, Shulman LH, Kennedy RF, et al. Idiopathic toe-walking: a review. Dev Med Child Neurol 1999:41:846-8.

5 Eastwood DM, Menelaus MB, Dickens DR, et al. Idiopathic toe-walking: does treatment alter the natural history? J Pediatr Orthop B 2000;9:47-9.

6 Hollister JR. Delay in motor development as a presentation of juvenile rheumatoid arthritis. J Pediatr 1981;15:581-3.

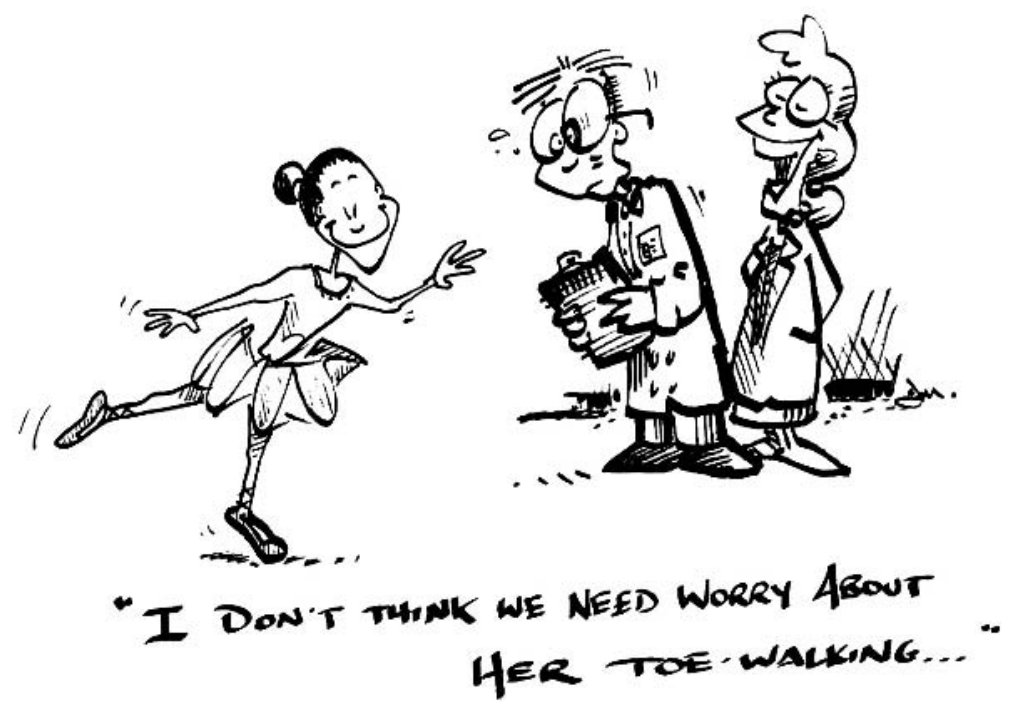

Illustration by Jack Maypole, MD 